Manuscript for Orbital2001

\title{
Absence of X-point Band Overlap in Divalent Hexaborides and Variability of the Surface Chemical Potential
}

\author{
J.D Denlinger, G.-H. Gweon ${ }^{1}$, S.-K. Mo ${ }^{1}$, J.W. Allen ${ }^{1}$, \\ J.L. SARRAO ${ }^{2}$, A.D. BIANCHI ${ }^{2}$, and Z. FisK ${ }^{3}$ \\ Advanced Light Source, Lawrence Berkeley National Laboratory, Berkeley CA 94720, USA \\ ${ }^{1}$ Randall Laboratory of Physics, University of Michigan, Ann Arbor, MI 48109-1120, USA \\ ${ }^{2}$ Los Alamos National Laboratory, Los Alamos, NM 87545 USA \\ ${ }^{3}$ National High Magnetic Field Lab and Dept. of Physics, Florida State University, Tallahassee, FL 32306, USA
}

\begin{abstract}
Angle-resolved photoemission measurements of divalent hexaborides reveals a $>1 \mathrm{eV} \mathrm{X}$-point gap between the valence and conduction bands, in contradiction to the band overlap assumed in several models of their novel ferromagnetism. While the global ARPES band structure and gap size observed are consistent with the results of bulk-sensitive soft x-ray absorption and emission boron K-edge spectroscopy, the surface-sensitive photoemission measurements also show a variation with cation, surface and time of the position of the surface chemical potential in the band structure.
\end{abstract}

KEYWORDS: hexaboride, $\mathrm{CaB}_{6}$, $\mathrm{EuB}_{6}$, angle resolve photemission

\section{§1. Introduction}

Great interest in the divalent hexaborides has been generated recently by the discovery of high Curie temperature weak-moment ferromagnetism (FM) in La-doped $\mathrm{CaB}_{6}{ }^{1)}$ and by exotic theoretical models to explain the unusual magnetism, e.g. that it represents the ground state of a dilute electron gas $^{2)}$ or of a doped excitonic insulator. ${ }^{3)}$ The starting point of most thinking about the divalent hexaborides, and central to the excitonic instability model, is the presumed existence of a band overlap between the top of the boron valence states and the bottom of the cation $d$-conduction band at the $\mathrm{X}$ point of the simple cubic Brillouin zone appropriate to these materials. Without such overlap stoichiometric divalent hexaborides would be insulators. Band overlap is predicted by band structure calculations ${ }^{4,5)}$ and de Haas van Alphen (dHvA) and Shubnikov de Hass (ShD) data ${ }^{6)}$ have been interpreted in this semi-metal framework.

Our early angle-resolved photoemission spectroscopy (ARPES) studies of $\mathrm{EuB}_{6}$ and $\mathrm{SrB}_{6}$ synchrotron measurements predating the FM discovery, showed, contrary to the band-overlap picture, an isolated $\mathrm{X}$-point electron pocket separated from the $\mathrm{X}$-point boron valence band maximum by a gap $>1 \mathrm{eV}$. However, motivated by the prior theory results, by apparently firm evidence for bulk band overlap from dHvA and $\mathrm{SdH}$ data discussed below and by certain surface sensitive aspects of our ARPES data, we interpreted ${ }^{7)}$ the observed gap as a property only of the surface region probed in ARPES. It was reported essentially simultaneously ${ }^{8}$ that a new band calculation including a GW self energy predicts $\mathrm{CaB}_{6}$ to have an X-point bandgap of $0.8 \mathrm{eV}$, similar to what we measured, thereby motivating us to reexamine our early
ARPES results as showing a bulk property. ${ }^{9)}$

We have recently presented new ARPES measurements and complementary bulk-sensitive soft x-ray emission and absorption ${ }^{9,10)}$ that demonstrate conclusively the existence of a bulk band gap at the X-point and a global band structure in good agreement with the GW band calculation. This experimental result rules out all mechanisms for FM requiring an X-point band overlap and also forces the consideration of boron vacancies as the origin of the conduction band carriers observed in nominally stoichiometric divalent material, and possibly $^{11)}$ also the origin of the magnetic moments.

It is important that ARPES data showing only a small conduction band pocket lying above the $\mathrm{X}$-point gap be reconciled with $\mathrm{dHvA}$ and $\mathrm{SdH}$ data on $\mathrm{EuB}_{6}$ implying two pieces of Fermi surface ${ }^{6)}$ interpreted as the electron and hole pockets of the X-point overlap picture. We have realized that the latter can also be interpreted as showing only a conduction band pocket, but spin split by a combination of the internal field of the Eu moments augmented by the large applied magnetic fields used for the measurement, rather than the electron and a hole pockets associated with X-point band overlap. Consistent with this new interpretation, more recent $\mathrm{dHvA}$ data for other divalent hexaborides ${ }^{12}$ ) show only a single piece of Fermi surface. We also note that the sign of the Hall effect is always negative. From all these findings we conclude that a bulk X-point band gap exists and that the bulk Fermi level $\left(E_{\mathrm{F}}\right)$ is typically near the bottom of the conduction band due to off-stoichiometry.

Ref. 9 summarizes without detail certain surface sensitive aspects of the ARPES data that are best interpreted as showing that, even though the bulk chemical potential appears typically to lie near the bottom of the conduc- 
tion band, the intersection of the chemical potential with the band structure at the surface can vary with cleave, with cation, with surface location and with time. In this paper we present the details of these observations and discuss their possible origins.

\section{§2. Experimental}

Single crystal samples of $\mathrm{CaB}_{6}, \mathrm{SrB}_{6}$ and $\mathrm{EuB}_{6}$ were grown from an aluminum flux using powders prepared by boro-thermally reducing cation oxides, a method shown to yield high quality with regard to both structure and chemical composition. ${ }^{13)}$ ARPES experiments were performed at the undulator beamline 10.0.1 of the Advanced Light Source synchrotron using a Scienta SES-200 electron spectrometer. Samples oriented by Laue diffraction were cleaved in situ to reveal a [100] surface just before the measurement, which was done at a sample temperature of $20-30 \mathrm{~K}$ and in a vacuum of $\approx 4 \times 10^{-11}$ Torr. A photon energy of $30 \mathrm{eV}$ was used to probe the $\Gamma-\mathrm{X}$ band structure, a value internally consistent with an "inner potential step" of $11.2 \mathrm{eV}$ experimentally determined for $\mathrm{EuB}_{6}$ from photon energy dependent measurements. The Fermi energy and instrumental resolution were calibrated with a reference spectrum taken on scraped $\mathrm{Au}$ or sputtered $\mathrm{Pt}$ foils. The ALS instrumental resolution was $22 \mathrm{meV}$ with total angular resolution of $0.3^{\circ}$. The angular detection mode of the Scienta analyzer, facilitating the simultaneous measurement of the complete band dispersions at the $\mathrm{X}$-point, was crucial to the monitoring of time-dependent changes in $\mathrm{EuB}_{6}$ presented below.

\section{$\S 3 . \quad$ Results}

Figure 1 shows the experimentally measured band structure for $\mathrm{CaB}_{6}$ along $\Gamma-\mathrm{X}$ with comparison to the GW band calculation. ${ }^{8)}$ The data, shown with reverse grayscale intensities, is the sum of two data sets with s- and p-polarization geometries that individually exhibit strong symmetry selection rule effects that we will present and analyze elsewhere.

The qualitative agreement between experiment and theory dispersions in Fig. 1(c), most importantly including the existence of a band gap near $E_{\mathrm{F}}$, is very striking. The energy axis for the theory bands has been shifted by $-0.5 \mathrm{eV}$ for better visual alignment to the experimental data. Quantitative discrepancies, however, are also quite visible, including: $(i)$ the size of the band gap, which should be larger so that the experimental $E_{\mathrm{F}}$ lies either below the conduction band or so near its minimum that its occupation is too small for detection by ARPES, (ii) the overlap between bands 2 and 3, (iii) the overall boron-block bandwidth (between bands 1 and 6 ), and $(i v)$ an additional weak broad dispersion at the bottom of the valence band. This counter-dispersing band, $11 \mathrm{eV}$ at $\Gamma$ to $\approx 9 \mathrm{eV}$ at $\mathrm{X}$ is suggestive of band backfolding resulting from a periodicity doubling. Indeed, low energy electron diffraction (LEED) images of freshly cleaved $\mathrm{CaB}_{6}$ surfaces exhibit $2 \times 1$ surface order as shown in Fig 2(a).
The absence of conduction band intensity at $E_{\mathrm{F}}$ for $\mathrm{CaB}_{6}$ in Fig. 1 implies a $>1 \mathrm{eV}$ band gap and insulating behavior. While this result agrees with electron counting for stoichiometric material, it does not agree with bulk Hall transport measurements of electron carriers $^{14)}$ and dHvA measurement of Fermi surfaces. ${ }^{6)}$ Also, as noted above, earlier ARPES measurements of EuB 6 and $\mathrm{SrB}_{6}$ both showed X-point electron pockets and ellipsoidal Fermi surface contours, ${ }^{7)}$ contrary to this $\mathrm{CaB}_{6}$ data set. Another important difference is the surface order revealed by LEED, that $\mathrm{CaB}_{6}$ shows a $2 \times 1$ surface reconstruction, whereas both $\mathrm{EuB}_{6}$ (Fig. 2(b)) and $\mathrm{SrB}_{6}$ both show only $1 \times 1$ order corresponding to the simple cubic lattice parameter. One possibility is that the $2 \times 1$ reconstruction in $\mathrm{CaB}_{6}$ helps to stabilize a surface with $E_{\mathrm{F}}$ in the band gap, although at the moment we have no specific mechanism to offer whereby this would be accomplished.

To further elucidate these different results for different cations, we present in Fig. 3 new ARPES measurements along $\Gamma$-X for $\mathrm{EuB}_{6}$ for the p-polarization geometry. Similar to the $\mathrm{CaB}_{6}$ data in Fig. 1(a), the $\Delta_{5}$ symmetry bands (2 and 5 ) are absent and no states near $E_{\mathrm{F}}$ are observed (see top panels of Fig. 3). However, this surface exhibited a very interesting time dependent behavior, shown in Fig. 3(b,c), in which the emergence of a small electron pocket at the $\mathrm{X}$-point is observed $\approx 4$ hours after cleavage of the sample. Accompanying this change at $E_{\mathrm{F}}$, some additonal redistribution of spectral weight in the boron block bands also occurs (illustrated by a comparison of X-point spectra in Fig. 3(c)).

The mechanism for this temporal change of the position of the chemical potential in the electronic structure observed by ARPES is not yet understood. However, such a dramatic shift of the chemical potential cannot occur in the entire bulk, and so we conclude that the mechanism must involve band bending that occurs from the bulk to the surface. If in the bulk the chemical potential lies in the gap, a possible scenario would be the creation of a metallic two-dimensional electron gas confined to the surface by downward bending (from bulk to surface) of the bottom of the conduction band as observed in $\mathrm{Cs} / \mathrm{n}-\operatorname{InAs}(110) .{ }^{15)}$ However, as mentioned above, the bulk transport, optics and dHvA measurements point clearly to the chemical potential lying in the conduction band for the bulk. We conclude therefore that upward band bending (from bulk to surface) of the bottom of the conduction band immediately after the cleave confines electrons to the sample interior, giving an initially insulating surface layer which then relaxes with time to a more bulk-like situation with the chemical potential at the surface lying at the bottom of the conduction band. We note that, while the surface order before and after the spectral changes in this particular sample is not known, only $1 \times 1$ LEED patterns, stable with time, have ever been observed for other freshly cleaved $\mathrm{EuB}_{6}$ surfaces.

One might expect the initial cleaved hexaboride surface to be metastable since either cation or boron termi- 
nation produces a polar (001) surface. In order for the two surfaces created by cleavage to be the same, there should be a statistical $50 \%$ cation termination on each surface. While the nature of the $2 \times 1$ resconstruction for $\mathrm{CaB}_{6}$ is not currently known, such a reconstruction appears to be natural considering these metastability arguments. One might speculate that due to a smaller ionic radius $\mathrm{Ca}$ atoms are more mobile than the $\mathrm{Eu}$ atoms so that the energy barrier for surface reconstruction is overcome for $\mathrm{CaB}_{6}$ even at the $20-30 \mathrm{~K}$ cleavage temperatures. On the other hand, for $\mathrm{EuB}_{6}$ the adsorption of residual gases may be required to induced the $\mathrm{EuB}_{6}$ surface to undergo a change from an initially metastable state (but apparently without a reconstruction) to a more bulk-like position of $E_{\mathrm{F}}$ at the surface. Total energy calculations of various surface terminations would be a first step to understanding these surface order effects.

Another likely example of band bending leading to surface dependence of the chemical potential position is a one time observation in a cation-rich $\mathrm{CaB}_{6}$ sample, designated $\mathrm{Ca}_{1+\delta} \mathrm{B}_{6}$, in which the chemical potential is observed to be located at the bottom of the band gap, actually cutting into the top of the boron valence bands, rather than in or close to the conduction band minimum. Data for this unusual sample are presented in Fig. 4 using two different polarization geometries. For the p-polarization data of $\mathrm{Ca}_{1+\delta} \mathrm{B}_{6}$ in Fig. 4(a), we first note that all energy bands are shifted to higher energy (smaller binding energy) by $\approx 1 \mathrm{eV}$ relative to $\mathrm{CaB}_{6}$ in Fig. 1. For example, the high intensity point at $\Gamma$ is shifted from $\approx 8 \mathrm{eV}$ to $\approx 7 \mathrm{eV}$, and the energy of the crossover of bands 2 and 3 is shifted from $\approx 3.3 \mathrm{eV}$ to $\approx 2.3 \mathrm{eV}$.

The s-polarization of the data in Fig. 4(b), highlights band 2 while suppressing band 3 . We observed two parallel dispersing bands, both of which we associate with band 2 and a large shallow hole pocket feature centered at $\mathrm{X}$, which we associate with band 1 dispersing through $E_{\mathrm{F}}$ at $k_{\|} \approx 0.3 \AA^{-1}$. We interpret the two-parallel dispersing parabolic bands as an indication either of lateral or depth inhomogeneity, or of excitation of two slightly misoriented facets. One set of $\mathrm{GW}$ bands shifted $+0.5 \mathrm{eV}$ from the original calculation is plotted in Fig. 4(a,b) and a second set of GW bands shifted $+1.4 \mathrm{eV}$ is also plotted in Fig. 4(b) to align with the upper dispersion. The surface areas of excitation are not necessarily the same in the two experimental geometries. We note that LEED on other samples shows regions of dual spots where the excitation hits two distinct facets that have slightly different surface normals. Cutting through different $k$-points on a second facet might explain the second energy shifted band.

This result points to a deficiency of electrons and holelike conduction in the surface region being probed. This can be achieved by $\mathrm{Ca}$ vacancies or excess boron atoms, which is contrary to the starting non-stoichiometric ratio of excess $\mathrm{Ca}$ in this $\mathrm{Ca}_{1+\delta} \mathrm{B}_{6}$ sample. While bulk trans- port on $\mathrm{Ca}$-rich $\mathrm{CaB}_{6}{ }^{16)}$ does exhibit low-energy scale differences compared to that of $\mathrm{CaB}_{6}$, e.g. semiconducting resistivity, we note that a separate ARPES experiment on $\mathrm{Ca}_{1+\delta} \mathrm{B}_{6}$ shows indistiguishable behavior from $\mathrm{CaB}_{6}$, i.e. no $\mathrm{X}$-point electron pocket and $>1 \mathrm{eV}$ band gap. Also, bulk-sensitive x-ray absorption and emission experiments show nearly identical spectra for $\mathrm{Ca}_{1+\delta} \mathrm{B}_{6}$ and $\mathrm{CaB}_{6}$ with no evidence of a large $1 \mathrm{eV}$ shift between their chemical potentials. Thus it seems that this one time finding with ARPES again signals band bending and variability in the position of the surface chemical potential different from the bulk.

\section{§4. Summary}

In summary, angle-resolved photoemission, in conjunction with soft X-ray absorption/emission spectroscopy presented elsewhere, ${ }^{9,10)}$ establishes the existence of an X-point bandgap (rather than band overlap) in the divalent hexaborides. Various bulk sensitive studies imply that the chemical potential typically lies near the bottom of the conduction band in the bulk, probably due to boron vacancies. However, ARPES also shows that the surface chemical potential position varies with cation, surface and time, implying band bending effects. In general it appears that the surface chemical potential position is relatively easy to vary, although not presently in a planned way. Ultimately this may be of practical importance if these materials have applications in spin-tronics devices, as has been proposed. ${ }^{8)}$

\section{Acknowledgements}

This work was supported at U. of Michigan by the U.S. DoE under Contract No. DE-FG02-90ER45416 and by the U.S. NSF Grant No. DMR-99-71611. The NHMFL is supported by the U.S. NSF Grant No. DMR-99-71348. The ALS is supported by the U.S. DoE under Contract No. DE-AC03-76SF00098.

1) D. P. Young et al., Nature 397, 412 (1999).

2) D. Ceperley, Nature 397, 386 (1999);G. Ortiz, M. Harris and P. Ballone, Phys. Rev. Lett. 82, 5317 (1999).

3) M. E. Zhitomirsky, T. M. Rice, and V. I. Anisimov, Nature 402, 251 (1999); L. Balents and C. M. Varma, Phys. Rev. Lett. 84, 1264 (2000); V. Barzykin and L. P. Gor'kov, Phys. Rev. Lett. 84, 2207 (2000).

4) A. Hasegawa and A. Yanase, J. Phys. C, Solid State Phys. 12, 5431 (1979).

5) S. Massidda, A. Continenza, T. M. D. Pascale, and R. Monnier, Z. Phys. B 102, 83 (1997).

6) R. G. Goodrich et al., Phys. Rev. B 58, 14896 (1998); M. C. Aronson et al., Phys. Rev. B 59, 4720 (1999).

7) J. D. Denlinger et al., cond-mat/0009022.

8) H. J. Tromp et al., Phys. Rev. Lett. 87, 016401 (2001).

9) J. D. Denlinger, J. A. Clack, J. W. Allen, G.-H. Gweon, D. M. Poirier, C. G. Olson, J. L. Sarrao, A. D. Bianchi and Z. Fisk, cond-mat/0107429.

10) J. D. Denlinger, G.-H. Gweon, J. W. Allen, A. D. Bianchi and Z. Fisk, cond-mat/0107426.

11) R. Monnier and B. Delley, Phys. Rev. Lett. 87, 157204 (2001).

12) D. Hall et al., cond-mat/0104184.

13) H. R. Ott et al., Z. Phys. B 102, 337 (1997). 
14) Z. Fisk et al., J. Appl. Phys. 50, 1911 (1979).

15) V. Yu Aristov et al., Phys. Rev. B 60, 7752 (1999).

16) P. Vonlanthen et al., Phys. Rev. B 62, 10076 (2000).

\section{$\S 5 . \quad$ Figures}

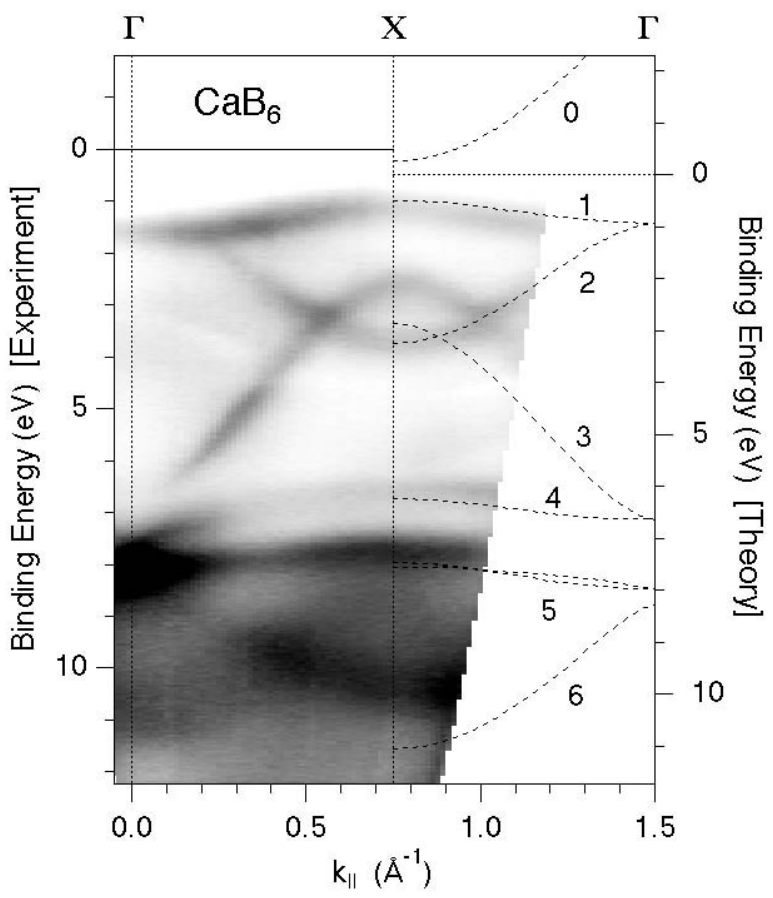

Fig.1. Comparison of the experimental and theoretical band structures of $\mathrm{CaB}_{6}$ along $\Gamma$-X. The reverse gray scale image of angle-resolved photoelectron intensities is the sum of two data sets with $30 \mathrm{eV} \mathrm{s}$ - and p-polarized excitation. Dashed lines are from the quasiparticle GW calculation ${ }^{8)}$ with its energy axis shifted by $0.5 \mathrm{eV}$ to lower energy.

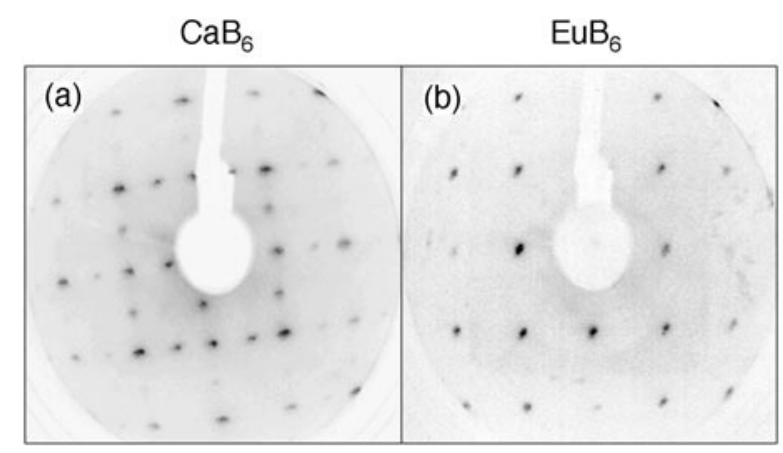

Fig. 2. Low energy electron diffraction images of freshly cleaved surfaces of (a) $\mathrm{CaB}_{6}$ and (b) $\mathrm{EuB}_{6}$ exhibiting $2 \times 1$ and $1 \times 1$ surface order, respectively. The beam energy is $165 \mathrm{eV}$ for both images.

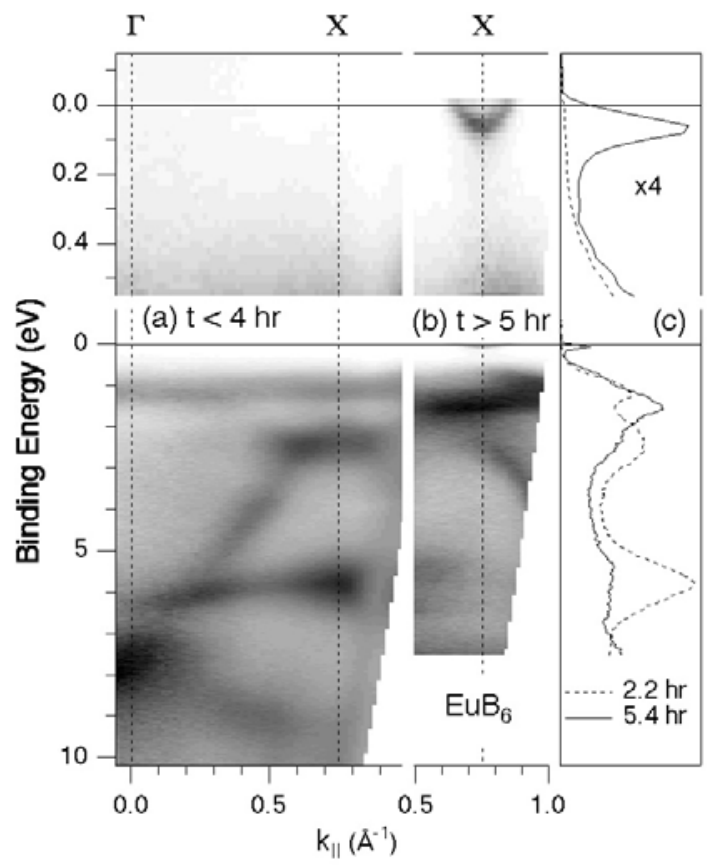

Fig. 3. Time dependent change in the band structure of $\mathrm{EuB}_{6}$. (a) $<4$ hours and(b) $>5$ hours after the initial cleave. The reverse gray scale images are measured with p-polarized $30 \mathrm{eV}$ excitation. (c) Comparison of EDCs from the X-point. Upper panels show an expanded region near $E_{\mathrm{F}}$.

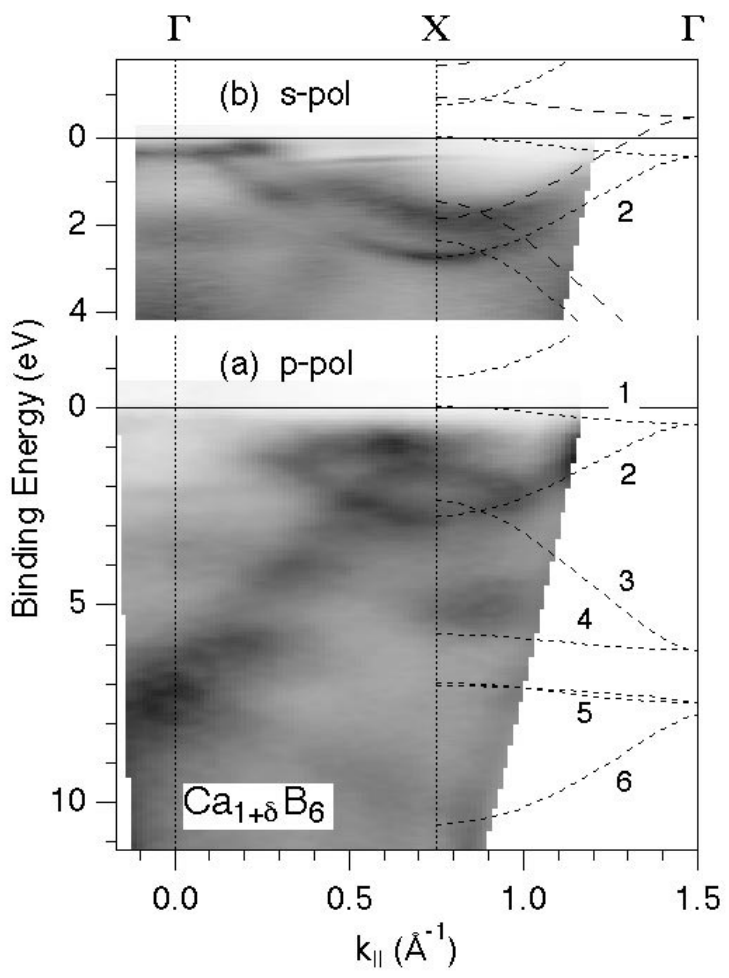

Fig. 4. ARPES band structure of $\mathrm{Ca}_{1+\delta} \mathrm{B}_{6}$ using (a) p-polarized and (b) s-polarized $30 \mathrm{eV}$ excitation. Dashed lines show the GW calculated bands shifted by 0.5 and $1.4 \mathrm{eV}$ to higher energy. 